\title{
ON FOURIER COEFFICIENTS OF MODULAR FORMS
}

\author{
C. J. CUMMINS ${ }^{\bowtie}$ and N. S. HAGHIGHI
}

(Received 2 December 2009)

\begin{abstract}
Recursive formulae satisfied by the Fourier coefficients of meromorphic modular forms on groups of genus zero have been investigated by several authors. Bruinier et al. ['The arithmetic of the values of modular functions and the divisors of modular forms', Compositio Math. 140(3) (2004), 552-566] found recurrences for $\operatorname{SL}(2, \mathbb{Z})$; Ahlgren ['The theta-operator and the divisors of modular forms on genus zero subgroups', Math. Res. Lett. 10(5-6) (2003), 787-798] investigated the groups $\Gamma_{0}(p)$; Atkinson ['Divisors of modular forms on $\Gamma_{0}(4)$ ', J. Number Theory 112(1) (2005), 189-204] considered $\Gamma_{0}(4)$, and S. Y. Choi ['The values of modular functions and modular forms', Canad. Math. Bull. 49(4) (2006), 526535] found the corresponding formulae for the groups $\Gamma_{0}^{+}(p)$. In this paper we generalize these results and find recursive formulae for the Fourier coefficients of any meromorphic modular form $f$ on any genuszero group $\Gamma$ commensurable with $\operatorname{SL}(2, \mathbb{Z})$, including noncongruence groups and expansions at irregular cusps. The form of the recurrence relations is well suited for the computation of the Fourier coefficients of the functions and forms on the groups which occur in monstrous and generalized moonshine. The required initial data has, in many cases, been computed by Norton (private communication).
\end{abstract}

2010 Mathematics subject classification: primary 11F30; secondary 11F12, 11F03.

Keywords and phrases: Fourier coefficients, modular forms, genus zero, moonshine, Hauptmodul.

\section{Introduction}

Suppose that $\Gamma$ is a genus-zero subgroup of $\operatorname{SL}(2, \mathbb{R})$ commensurable with $\operatorname{SL}(2, \mathbb{Z})$. Let

$$
f=q^{r}+\sum_{n=1}^{\infty} a_{n} q^{r+n},
$$

be a meromorphic modular form of integer weight $k$ on $\Gamma$, where $q$ is a suitable local parameter at the cusp $\infty$.

When $\Gamma=\operatorname{SL}(2, \mathbb{Z})$, the problem of finding universal recursive relations satisfied by the Fourier coefficients of $f$ was investigated by Bruinier et al. in [6].

Shortly after this work, Ahlgren in [1] and Atkinson in [3] obtained similar results for the cases $\Gamma_{0}(p)(p=2,3,5,7,13)$ and $\Gamma_{0}(4)$, respectively. S. Y. Choi

This work was supported in part by NSERC.

(C) 2010 Australian Mathematical Publishing Association Inc. 0004-9727/2010 \$16.00 
in [8] considered the case $\Gamma_{0}^{+}(p)$, which is the group $\Gamma_{0}(p)$ extended by the Fricke involution. These groups have genus zero for $p \in\{2,3,5,7,11,13,17,19$, 23, 29, 31, 41, 47, 59, 71\}. S. Y. Choi also gave recursive relations expressing the coefficients of a Hauptmodul $j_{1}$ of $\Gamma_{1}$ by using those of the normalized Hauptmodul $j_{2}$ of $\Gamma_{2}$, where $\Gamma_{1}$ is a Fuchsian group of the first kind of genus zero and $\Gamma_{2}$ is a finite-index, genus-zero subgroup of $\Gamma_{1}$. In related work, D. Choi [7] considered the case of forms on $\Gamma_{0}(N)$ for which $N$ is square-free, but not necessarily genus zero, in terms of certain ' $(\ell, N)$-type' sequences of modular functions.

The strategy of these papers, following that of [6], has been to express $(\theta f) / f$ in terms of a modular form $f_{\theta}$ of weight 2 and terms involving the Eisenstein series $E_{2}$, where $\theta$ is the Ramanujan $\theta$ operator, and then to compute $(1 / 2 \pi i) \int f_{\theta}(z) F_{n}(z) d z$, along the boundary of a fundamental domain of the group in question, where $F_{n}$, $n=1,2,3, \ldots$ are a certain polynomials in the Hauptmodul of $\Gamma$ known as the Faber polynomials.

The motivation for the present paper is to generalize this work, using a somewhat different method, so as to facilitate and simplify the computations of the Fourier coefficients of the modular functions and forms which occur in moonshine and generalized moonshine [9].

In this case, the groups of interest are discrete, genus zero, contain some $\Gamma_{0}(N)$, and the subgroup which stabilizes $\infty$ is precisely the subgroup of translations by integers. We shall call these groups 'moonshine type' groups. In the case of monstrous moonshine the groups are between some $\Gamma_{0}(N)$ and its normalizer in $\operatorname{SL}(2, \mathbb{R})$, but for generalized moonshine the inclusion is not necessarily normal; see [9].

Several types of recurrence relations for the Hauptmoduls which occur in moonshine are known. For example, in his proof of the monstrous moonshine conjectures, Borcherds [4] makes use of certain recurrence formulae which generalize those found by Lehmer [17] and Mahler [19]. For a generalization of Mahler's recurrences to some Hauptmoduls with irrational coefficients, see [12].

Another type of recurrence was introduced by Norton [22], who defined the notion of a replicable function. This gives rise to a new set of recurrence relations for Hauptmoduls with rational coefficients on moonshine type groups. Conjecturally, these recurrences can be extended to the case of Hauptmoduls with irrational coefficients on moonshine type groups. For more information on replicable functions see, for example, [2, 9, 13, 22].

More generally, a tabulation of all $\operatorname{SL}(2, \mathbb{R})$ conjugacy classes of congruence subgroups of genus zero and of genus one was found in [10, 11]. The results are that there are $504 \operatorname{SL}(2, \mathbb{R})$ conjugacy classes of genus-zero, congruence subgroups. These give rise to 616 groups whose Hauptmoduls have rational coefficients and which contain some $\Gamma_{0}(N)$. Conjecturally, these Hauptmoduls correspond precisely to Norton's rational replicable functions (with the exception of three trivial cases).

The recurrence formulae we give in this paper have the virtue of applying uniformly to any group of genus zero which is commensurable with the modular group. In particular, they apply to moonshine type groups. They also apply to the computation 
of the coefficients of modular forms, not only Hauptmoduls. The required input is certain ramification data. For moonshine type groups with rational Hauptmoduls, the ramification data has been calculated by Norton [23]. Thus, for these cases, the recurrences we give are of immediate utility. It would be very interesting to complete this computation to include all 504 cases of conjugacy classes of genus-zero groups found in [10].

To be more precise, let $\Gamma_{0}^{+}(N)$ denote the group $\Gamma_{0}(N)$ extended by all its AtkinLehner elements. Then the recurrence relations we find have as input a square-free integer $N$ for which $\Gamma$ is conjugate to a subgroup of $\Gamma_{0}^{+}(N)$. This is possible by a theorem of Helling [14]. Also required are the ramification locus and ramification orders of a normalized Hauptmodul $\phi_{\Gamma}$ of $\Gamma$. Then, given the weight and divisor of $f$ and the values of $\phi_{\Gamma}$ at the points at which the divisor of $f$ is supported, the recurrence relations for the Fourier coefficients of $f$ are given in Theorems 3.6 and 3.7. These have the same form for all $\Gamma$ and $f$, and in this sense are universal.

Since the 'Helling groups', $\Gamma_{0}^{+}(N)$ are transitive on cusps, the form of the recurrence relations at any cusp of $\Gamma$ is once again given by Theorems 3.6 and 3.7. Although our motivation was to find algorithms for computing the Fourier coefficients of functions and forms for groups of moonshine type, the results hold for any genuszero group commensurable with the modular group, including irregular groups (those which do not contain $-\mathbf{1}_{2}$ ) and noncongruence subgroups. For the irregular groups, expansions at irregular cusps may be computed.

\section{Preliminaries}

Let $\Gamma$ be a finite-index subgroup of $\Gamma_{0}^{+}(N)$, for some square-free $N . \Gamma_{0}^{+}(N)$ as well as $\Gamma$ act on $\mathcal{H}^{*}$ by Möbius transformations, where $\mathcal{H}^{*}$ is the union of the upper halfplane $\mathcal{H}$ with the projective rational line $\mathbb{P}_{1}(\mathbb{Q})$. Assume that $s_{0}=\infty, s_{1}, \ldots, s_{t} \in$ $\mathbb{P}_{1}(\mathbb{Q})$ are a set of representatives of $\Gamma \backslash \mathbb{P}_{1}(\mathbb{Q})$.

Since $\Gamma_{0}^{+}(N)$ acts transitively on $\mathbb{P}_{1}(\mathbb{Q})$ (see [15]), there is only one equivalence class of cusps and this class contains $\infty$. The stabilizer subgroup of $\infty$ in $\Gamma_{0}^{+}(N)$ is

$$
\left\{ \pm\left(\begin{array}{ll}
1 & n \\
0 & 1
\end{array}\right): n \in \mathbb{Z}\right\} .
$$

Now we fix a cusp $s$ of $\Gamma$ and then take an element $\gamma \in \Gamma_{0}^{+}(N)$ such that $\gamma(s)=\infty$. We have

$$
\pm \gamma \Gamma_{s} \gamma^{-1}=\left\{ \pm\left(\begin{array}{cc}
1 & h \\
0 & 1
\end{array}\right)^{m}: m \in \mathbb{Z}\right\},
$$

where $h$ is a positive integer and $\Gamma_{s}:=\{\alpha \in \Gamma: \alpha(s)=s\}$ is the stabilizer subgroup of $s$ in $\Gamma$. If $-\mathbf{1}_{2} \notin \Gamma$, then $\gamma \Gamma_{s} \gamma^{-1}$ is generated either by $\left(\begin{array}{ll}1 & h \\ 0 & 1\end{array}\right)$ or by $\left(\begin{array}{rr}-1 & h \\ 0 & -1\end{array}\right)$. The cusp $s$ is called regular or irregular of width $h$ accordingly. By the definition, if $-\mathbf{1}_{2} \in \Gamma$, then all the cusps of $\Gamma$ are regular. We call such a group regular. If $-\mathbf{1}_{2}$ is not in $\Gamma$, then we call $\Gamma$ irregular. Thus regular groups have only regular cusps, while irregular groups can have regular or irregular cusps or a mixture of the two. 
For a subgroup $G$ of $\operatorname{SL}(2, \mathbb{R})$, we define $\bar{G}$ to be the image of $G$ in $\operatorname{PSL}(2, \mathbb{R})=$ $\operatorname{SL}(2, \mathbb{R}) /\{ \pm 1\}$. If $h_{0}, h_{1}, \ldots, h_{t}$ are the cusp widths of the cusps $\infty, s_{1}, \ldots, s_{t}$ of $\Gamma$, then the following relation holds (see [16]):

$$
\sum_{i=0}^{t} h_{i}=\left[\overline{\Gamma_{0}^{+}(N)}: \bar{\Gamma}\right]
$$

This is called the cusp-split equation of $\Gamma$.

A complex-valued function $f$ on the upper half-plane $\mathcal{H}$ is said to be a meromorphic (respectively, holomorphic) modular function of weight $k$ with respect to $\Gamma$ if:

- $\quad f$ is meromorphic (respectively, holomorphic) on $\mathcal{H}$;

- $\quad f \mid[\rho]_{k}=f$, for all $\rho=\left(\begin{array}{ll}a & b \\ c & d\end{array}\right) \in \Gamma$, where $f \mid[\rho]_{k}(z):=(c z+d)^{-k} f(\rho(z))$;

- $\quad f$ is meromorphic (respectively, holomorphic) at every cusp $s$ of $\Gamma$.

This last condition means that

$$
f \mid\left[\gamma^{-1}\right]_{k}(z)= \begin{cases}\Psi\left(e^{\pi i z / h}\right) & \text { if } k \text { is odd and } s \text { is irregular } \\ \Phi\left(e^{2 \pi i z / h}\right) & \text { otherwise }\end{cases}
$$

where $\Phi$ and $\Psi$ are a meromorphic (respectively, holomorphic) functions at zero, $\Psi$ is an odd function, and $h$ and $\gamma$ are as in Equation (2.1). For more details, see [24, Definition 2.1].

We shall denote by $A_{k}(\Gamma)$ (respectively, $G_{k}(\Gamma)$ ) the space of meromorphic (respectively, holomorphic) modular forms of weight $k$ for $\Gamma$. Moreover, if for each cusp of $\Gamma$, the function $\Phi$ (or $\Psi$ ) in (2.3) vanishes at zero, then $f$ is called a cusp form on $\Gamma$, and the set of all such cusp forms will be denoted by $S_{k}(\Gamma)$.

Let us recall the definition of the order of a meromorphic function $f$ at a point $\tau \in \Gamma \backslash \mathcal{H}^{*}$. First, for $\tau$ corresponding to a point $z_{0} \in \mathcal{H}$, we set $e_{\tau}=\left|\bar{\Gamma}_{\tau}\right|$, where $\bar{\Gamma}_{\tau}$ is the stabilizer subgroup of $\tau$. Then $\operatorname{ord}_{\tau} f$ is defined (see [24, Section 2.4]) as

$$
\operatorname{ord}_{\tau} f:=e_{\tau}^{-1} \operatorname{ord}_{\left(z-z_{0}\right)} f \text {. }
$$

If $\tau$ corresponds to a cusp $s$ of $\Gamma$, with cusp width $h$, then

$$
\operatorname{ord}_{\tau} f:= \begin{cases}\left(\operatorname{ord}_{e^{\pi i z / h}} \Psi\right) / 2 & \text { if } k \text { is odd and s is irregular, } \\ \operatorname{ord}_{e^{2 \pi i z / h}} \Phi & \text { otherwise, }\end{cases}
$$

where $\Phi$ and $\Psi$ are as in (2.3). We can associate with each $f \in A_{k}(\Gamma)$ the divisor of $f$ defined as

$$
\operatorname{div}(f):=\sum_{\tau \in \Gamma \backslash \mathcal{H}^{*}} \operatorname{ord}_{\tau} f[\tau] .
$$

From now on, we suppose that $\Gamma$ is of genus zero, by which we mean that the compact Riemann surface $\Gamma \backslash \mathcal{H}^{*}$ is of genus zero. Any generator $\phi_{\Gamma}$ of the function field of this Riemann surface is called a Hauptmodul of $\Gamma$. When no confusion arises, 
we write $\phi$ rather than $\phi_{\Gamma}$. If we require $\phi$ to have a simple pole at the cusp infinity, then (see, for instance, [24, Ch. 2]) after normalization, $\phi$ has a $q$-expansion of the form

$$
\phi(q)=\frac{1}{q}+\sum_{n \geq 0} c_{n} q^{n}
$$

where $q=e^{2 \pi i z / h_{0}}$.

REMARK 2.1. In light of (2.3), any modular form $f(z)$ of weight $k$ may also be viewed as a function of $q$, namely $f(z)=\Phi(q)$ (or $f(z)=\Psi\left(q^{1 / 2}\right)$ ). Therefore, by an abuse of notation, we allow ourselves to write $f(q)$, viewing $f$ as a function of $q$. The same remark also applies to any reference to derivatives. In other words, $f^{\prime}(q)=d f / d q$ should be understood as $\left(h_{0} / 2 \pi i q\right)(d f / d z)$. So, from now on we suppress $\Phi$ and $\Psi$ from the notation.

2.1. Faber polynomials. Let

$$
\varphi(q)=\frac{1}{q}+\sum_{n \geq 0} a_{n}(\varphi) q^{n}
$$

be a formal power series in $q$. For $n \geq 1$, define the $n$th Faber polynomial to be the unique monic polynomial $F_{n}$ of degree $n$ which satisfies the relation

$$
F_{n}(\varphi)=\frac{1}{q^{n}}+\sum_{m \geq 1}^{\infty} F_{n, m} q^{m}
$$

The coefficients $F_{n, m}$ depend on the coefficients $a_{n}(\varphi)$. For later computations, it is also convenient to set $F_{0}=1$. Following this convention (see Relation 14 on page 35 of [25]), one may verify that the Faber polynomials are given by the formal generating series

$$
\frac{q \varphi^{\prime}(q)}{w-\varphi(q)}=\sum_{n=0}^{\infty} F_{n}(w) q^{n}
$$

where $\varphi^{\prime}(q)$ is the derivative of $\varphi$ with respect to $q$. In fact, (2.6) turns out to be an equality between holomorphic functions if $\phi$ is a holomorphic function on a bounded simply connected domain (for the details, see [25, Theorem 1, p. 51]). The Faber polynomials $F_{n}$, for $n \geq 0$, also satisfy the recurrence relation

$$
F_{n+1}(w)=w F_{n}(w)-\sum_{k=0}^{n} a_{n-k}(\varphi) F_{k}(w)-n a_{n}(\varphi) ;
$$

see, for instance, the relations in (7), page 40 of [25]. It is also possible to give an explicit formula for $F_{n}$ in terms of the coefficients $a_{0}(\varphi), a_{1}(\varphi), \ldots, a_{n-1}(\varphi), n \geq 1$. This can be done by invoking the following lemma whose proof has been inspired by relation (2.11) on page 23 and Example 20 on page 33 of [18]. 
LEMMA 2.2. Let $\Lambda=\mathbb{Q}\left[\alpha_{1}, \alpha_{2}, \alpha_{3}, \ldots\right]$, and set $\alpha_{0}=1$. Now suppose that $S_{i} \in \Lambda$ $(i=1,2,3, \ldots)$ are such that

$$
n \alpha_{n}+\sum_{j=1}^{n} S_{j} \alpha_{n-j}=0 \quad(\forall n \geq 1) .
$$

Then

$$
S_{n}=S\left(n ; \alpha_{1}, \ldots, \alpha_{n}\right)
$$

where

$$
\begin{aligned}
& S\left(l ; \alpha_{1}, \ldots, \alpha_{n}\right) \\
& :=l \sum_{\substack{m_{1}, \ldots, m_{n} \geq 0 \\
m_{1}+2 m_{2}+\cdots+n m_{n}=l}}(-1)^{m_{1}+\cdots+m_{n}} \frac{\left(m_{1}+\cdots+m_{n}-1\right) !}{m_{1} ! \cdots m_{n} !} \alpha_{1}^{m_{1}} \cdots \alpha_{n}^{m_{n}} .
\end{aligned}
$$

PROOF. In $\Lambda[[t]]$, let

$$
H(t)=\sum_{n \geq 0} \alpha_{n} t^{n} \quad \text { and } \quad P(t)=\sum_{n \geq 1} S_{n} t^{n-1} .
$$

Then

$$
-H(t) P(t)=H^{\prime}(t)
$$

This is true since

$$
\begin{aligned}
H^{\prime}(t) & =\sum_{n \geq 1} n \alpha_{n} t^{n-1} \\
& =\sum_{n \geq 1}\left(\sum_{j=1}^{n}-S_{j} \alpha_{n-j}\right) t^{n-1} \\
& =-\sum_{n \geq 1} S_{n} t^{n-1} \sum_{n \geq 0} \alpha_{n} t^{n} \\
& =-P(t) H(t) .
\end{aligned}
$$

For any element $z \in t \Lambda[[t]]$, define $L(z)=z+z^{2} / 2+z^{3} / 3+\cdots$. One has the identity $(1+z) L^{\prime}(-z)=-z^{\prime}$. Taking $z=H^{+}(t)=\sum_{n \geq 1} \alpha_{n} t^{n}$, this yields

$$
H(t)\left(H^{+}(t)-\frac{1}{2} H^{+}(t)^{2}+\frac{1}{3} H^{+}(t)^{3}-\cdots\right)^{\prime}=-P(t) H(t) .
$$

Thus, since $H(t)$ is not a zero divisor in $\Lambda[[t]]$,

$$
-P(t)=\left(H^{+}(t)-\frac{1}{2} H^{+}(t)^{2}+\frac{1}{3} H^{+}(t)^{3}-\cdots\right)^{\prime} .
$$

To compute the coefficient of $n t^{n-1}$ in (2.9) we argue as follows. For any choice of $m_{1}, \ldots, m_{n} \geq 0$ satisfying $m_{1}+\cdots+n m_{n}=n$, write $k=m_{1}+m_{2}+\cdots+m_{n}$ and 
note that $1 \leq k \leq n$. Such a choice uniquely gives rise to the term

$$
\begin{aligned}
& \frac{(-1)^{k-1} k !}{k m_{1} ! \cdots m_{n} !}\left(\alpha_{1} t\right)^{m_{1}}\left(\alpha_{2} t^{2}\right)^{m_{2}} \cdots\left(\alpha_{n} t^{n}\right)^{m_{n}} \\
& \quad=(-1)^{k-1} \frac{\left(m_{1}+\cdots+m_{n}-1\right) !}{m_{1} ! \cdots m_{n} !} \alpha_{1}^{m_{1}} \cdots \alpha_{n}^{m_{n}} t^{n},
\end{aligned}
$$

obtained by the generalized binomial theorem applied to the $k$ th term appearing in $H^{+}(t)-\frac{1}{2} H^{+}(t)^{2}+\frac{1}{3} H^{+}(t)^{3}-\cdots$. Now taking the sum over all possible choices gives the desired result.

Combining Lemma 2.2 with (2.7) yields the following formula for the Faber polynomials (cf. [5, Theorem 1.3]).

\section{COROllary 2.3.}

$$
\begin{aligned}
F_{n}(w)= & S\left(n ; a_{0}(\varphi)-w, a_{1}(\varphi), \ldots, a_{n-1}(\varphi)\right) \\
= & n \sum_{\substack{m_{1}+2 m_{2}+\cdots+n m_{n}=n\\
}}(-1)^{m_{1}+m_{2}+\cdots+m_{n}} \frac{\left(m_{1}+\cdots+m_{n}-1\right) !}{m_{1} ! \cdots m_{n} !} \\
& \times\left(a_{0}(\varphi)-w\right)^{m_{1}} a_{1}(\varphi)^{m_{2}} \cdots a_{n-1}(\varphi)^{m_{n}}
\end{aligned}
$$

\section{The recurrence relations}

In this section we find recurrence relations for forms on any genus-zero subgroup $\Gamma$ of $\operatorname{SL}(2, \mathbb{R})$ which is commensurable with $\operatorname{SL}(2, \mathbb{Z})$. As mentioned in the introduction, Helling in [14] proved the following result.

THEOREM 3.1. There exist a $\rho \in \mathbf{G L}_{2}{ }^{+}(\mathbb{Q})$ and a square-free integer $N$ such that

$$
\rho^{-1} \Gamma \rho \subseteq \Gamma_{0}^{+}(N)
$$

We remark that, as the map $f \mapsto f \mid[\rho]_{k}$ provides an isomorphism of complex vector spaces between $A_{k}(\Gamma)$ and $A_{k}\left(\rho^{-1} \Gamma \rho\right)$ (see, for example, [24, Proposition 2.4]), we may restrict ourselves to the case where $\Gamma$ is contained in $\Gamma_{0}^{+}(N)$. So, from now on, we assume that $\Gamma \subseteq \Gamma_{0}^{+}(N)$ for the same square-free $N$. Also, since $\Gamma_{0}^{+}(N)$ acts transitively on cusps, without loss of generality, we only need consider the cusp $\infty$.

Now let

$$
f(z)=q^{r}+\sum_{n=1}^{\infty} a_{n} q^{r+n} \in A_{k}(\Gamma)
$$

be a meromorphic modular form of weight $k$ for $\Gamma$, where $q=e^{2 \pi i z / h_{0}}$. As discussed in Section 2, if $k$ is even, or if $k$ is odd and the cusp $\infty$ is regular, then $r$ is an integer. While if $k$ is odd and the cusp $\infty$ is irregular, $r$ is equal to $r^{\prime} / 2$ for some odd integer $r^{\prime}$. 
3.1. The weight zero quotient. For a square-free integer $N$, let $v\left(\Gamma_{0}^{+}(N)\right)$ be the volume of the compact Riemann surface $\Gamma_{0}^{+}(N) \backslash \mathcal{H}^{*}$, which is given by

$$
v\left(\Gamma_{0}^{+}(N)\right)=\frac{\pi}{3} \prod_{p \mid N} \frac{p+1}{2} ;
$$

see, for example, [15, Section 3]. This formula, together with [24, Proposition 2.16, Theorem 2.20] and the cusp-split formula (2.2), implies that

$$
\sum_{\tau \in \Gamma \backslash \mathcal{H}^{*}} \operatorname{ord}_{\tau} f=\frac{k}{12} \prod_{p \mid N} \frac{p+1}{2} \sum_{i=0}^{t} h_{i} .
$$

Let $\eta(z)$ be the Dedekind eta function and $\Delta(z)=\eta^{24}(z)$. To continue, we seek a cusp form for $\Gamma_{0}^{+}(N)$ which plays the same role as $\Delta$ does for $\operatorname{SL}(2, \mathbb{Z})$. By the results of Newman [20,21], for some suitable $\ell$, the function

$$
h(z)=\prod_{\delta \mid N} \eta(\delta z)^{\ell}
$$

is a cusp form of weight $\varepsilon=(\ell / 2) \tau(N)$ on $\Gamma_{0}(N)$, where $\tau(N)$ is the number of positive divisors of $N$. Moreover, since $N$ is square-free, $\Gamma_{0}^{+}(N)$ is the normalizer of $\Gamma_{0}(N)$; see, for example, [15]. It follows that $h(z)$ is a form on $\Gamma_{0}^{+}(N)$. As we shall see, the results below are independent of the choice of $\ell$.

The form $h(z)$ is holomorphic and nonvanishing on $\mathcal{H}$ and has a zero of order $(\ell / 24) \sigma(N)$ at $\infty$, where $\sigma(N)=\sum_{\delta \mid N} \delta$. One can easily see that

$$
\Theta:=\frac{f^{\varepsilon /(\varepsilon, k)}}{h^{k /(\varepsilon, k)}}
$$

is a meromorphic modular form of weight zero on $\Gamma$, and therefore it can be expressed as a rational function of $\phi$, the generator of the function field of $\Gamma \backslash \mathcal{H}^{*}$. The first lemma of the next section gives the precise relation between $\Theta$ and $\phi$.

3.2. The recursive formulae. We first state and prove two preliminary results which provide us with enough ingredients for the proof of the main theorem of this section.

LEMMA 3.2. Let the notation be as above. Then, for some nonzero constant $\lambda$,

$$
\begin{aligned}
\Theta(z)=\lambda & \prod_{i=1}^{t}\left(\phi(z)-\phi\left(s_{i}\right)\right)^{\frac{\varepsilon}{(\varepsilon, k)} \operatorname{ord}_{s_{i}} f-\frac{\ell h_{i} k \sigma(N)}{24(\varepsilon, k)}} \\
& \times \prod_{\tau \in \Gamma \backslash \mathcal{H}}(\phi(z)-\phi(\tau))^{\frac{\varepsilon}{(\varepsilon, k)} \operatorname{ord}_{\tau} f} .
\end{aligned}
$$

PROOF. To prove this equality it is enough to verify that both sides have the same order for the points of $\Gamma \backslash \mathcal{H}^{*}$. Since $h(z)$ is holomorphic and nonvanishing on the upper half-plane, for any $\tau \in \Gamma \backslash \mathcal{H}$ the order of both sides of (3.3) is the same. For any cusp $s_{i}, 1 \leq i \leq t$, we also have the equality of the orders because $\operatorname{ord}_{s_{i}} h=\ell h_{i} \sigma(N) / 24$. 
At the cusp $\infty$ we start with the equality (3.2) and immediately we have

$$
\frac{\ell}{2} \tau(N) \sum_{\tau \in \Gamma \backslash \mathcal{H}^{*}} \operatorname{ord}_{\tau} f=\frac{\ell k}{24} \prod_{p \mid N}(p+1) \sum_{i=0}^{t} h_{i} .
$$

This relation, combined with the identity $\prod_{p \mid N}(p+1)=\sigma(N)$, implies that

$$
\frac{\varepsilon}{(\varepsilon, k)} \operatorname{ord}_{\infty} f-\frac{\ell h_{0} k \sigma(N)}{24(\varepsilon, k)}=\sum_{i=1}^{t} \frac{\ell h_{i} k \sigma(N)}{24(\varepsilon, k)}-\sum_{\tau \in \Gamma \backslash \mathcal{H}^{*}} \frac{\varepsilon}{(\varepsilon, k)} \operatorname{ord}_{\tau} f .
$$

The last relation shows the equality of the orders at $\infty$ and this completes the proof.

LEMMA 3.3. With the notation as above, the coefficients of $f$ satisfy the recursive relation

$$
a_{n}=\frac{-\beta_{1} a_{n-1}-\beta_{2} a_{n-2}-\cdots-\beta_{n-1} a_{1}-\beta_{n}}{n},
$$

where, for $n \geq 1, \beta_{n}$ is defined by the formula

$$
\begin{gathered}
\beta_{n}:=\frac{2 k h_{0}}{\tau(N)} \sum_{\delta \mid N} \delta \sigma\left(\frac{n}{\delta h_{0}}\right)+\sum_{i=1}^{t}\left(\operatorname{ord}_{s_{i}} f-\frac{k h_{i} \sigma(N)}{12 \tau(N)}\right) F_{n}\left(s_{i}\right) \\
+\sum_{\tau \in \Gamma \backslash \mathcal{H}} \operatorname{ord}_{\tau} f F_{n}(\tau) .
\end{gathered}
$$

Here $\sigma\left(n / \delta h_{0}\right)=0$, if $\delta h_{0} \nmid n$.

PROOF. Taking the logarithmic derivative of (3.3) and using Equation (2.6), we find

$$
\begin{aligned}
\frac{q f^{\prime}(q)}{f(q)}=\frac{\ell k h_{0}}{\varepsilon} & \sum_{\delta \mid N} \frac{\delta}{24} E_{2}\left(q^{\delta h_{0}}\right) \\
& -\sum_{i=1}^{t}\left(\left(\operatorname{ord}_{s_{i}} f-\frac{k h_{i} \sigma(N)}{12 \tau(N)}\right) \sum_{n=0}^{\infty} F_{n}\left(s_{i}\right) q^{n}\right) \\
& -\sum_{\tau \in \Gamma \backslash \mathcal{H}}\left(\operatorname{ord}_{\tau} f \sum_{n=0}^{\infty} F_{n}(\tau) q^{n}\right)
\end{aligned}
$$

where $F_{n}$ is the $n$th Faber polynomial associated with $\phi$ and $E_{2}(q)=1-$ $24 \sum_{n=1}^{\infty} \sigma(n) q^{n}$ is the nonmodular Eisenstein series of weight 2 . The last equality can be written as

$$
\frac{q f^{\prime}(q)}{f(q)}=\frac{\ell k h_{0} \sigma(N)}{24 \varepsilon}-\frac{k h_{0} \sigma(N)}{12 \tau(N)}+r-\sum_{n=1}^{\infty} \beta_{n} q^{n}=r-\sum_{n=1}^{\infty} \beta_{n} q^{n},
$$


where $\beta_{n}$ is defined as in (3.5). Substituting the $q$-expansion of $f$ given in (3.1) in Equation (3.7) yields

$$
r+\sum_{n=1}^{\infty}(r+n) a_{n} q^{n}=\left(1+\sum_{n=1}^{\infty} a_{n} q^{n}\right)\left(r-\sum_{n=1}^{\infty} \beta_{n} q^{n}\right) .
$$

By equating the coefficients of $q^{n}$ on both sides of this last equality,

$$
(r+n) a_{n}=r a_{n}-\beta_{1} a_{n-1}-\beta_{2} a_{n-2}-\cdots-\beta_{n},
$$

and this last equality yields the desired identity (3.4).

LEMMA 3.4. Keeping the assumptions as before,

$$
\beta_{n}=S\left(n ; a_{1}, \ldots, a_{n}\right),
$$

where $S\left(n ; a_{1}, \ldots, a_{n}\right)$ is defined as in Lemma 2.2.

PROOF. By comparing (3.4) and (2.8), we see that the $\beta_{n}, n=1,2, \ldots$, satisfy the same relations as the $S_{n}, n=1,2, \ldots$, and so the result follows by Lemma 2.2.

THEOREM 3.5. Let $f=q^{r}+\sum_{n=1}^{\infty} a_{n} q^{r+n}$ be a weight-k meromorphic modular form for the genus-zero subgroup $\Gamma$ of $\Gamma_{0}^{+}(N)$. Let $s_{0}=\infty, s_{1}, s_{2}, \ldots, s_{t}$ be representatives of the equivalence classes of cusps of $\Gamma$, of widths $h_{0}, h_{1}, h_{2}, \ldots, h_{t}$, respectively. Then

$$
\begin{aligned}
a_{n}= & \sum_{\substack{m_{1}, \ldots, m_{n-1} \geq 0 \\
m_{1}+\cdots+(n-1) m_{n-1}=n}}(-1)^{m_{1}+\cdots+m_{n-1}} \frac{\left(m_{1}+\cdots+m_{n-1}-1\right) !}{m_{1} ! \cdots m_{n-1} !} a_{1}^{m_{1}} \cdots a_{n-1}^{m_{n-1}} \\
& -\frac{1}{n}\left(\frac{2 k h_{0}}{\tau(N)} \sum_{\delta \mid N} \delta \sigma\left(\frac{n}{\delta h_{0}}\right)+\sum_{i=1}^{t}\left(\operatorname{ord}_{s_{i}} f-\frac{k h_{i} \sigma(N)}{12 \tau(N)}\right) F_{n}\left(\phi\left(s_{i}\right)\right)\right. \\
& \left.+\sum_{\tau \in \Gamma \backslash \mathcal{H}} \operatorname{ord}_{\tau} f F_{n}(\phi(\tau))\right),
\end{aligned}
$$

where $F_{n}$ is the nth Faber polynomial associated with the Hauptmodul $\phi$ of $\Gamma$.

PROOF. The result follows from Lemmas 3.3 and 3.4.

Theorem 3.5 gives recursive formulae for the coefficients of the modular form $f$. These formulae involve the Faber polynomials evaluated at points of $\mathcal{H}^{*}$. These polynomials can also be calculated recursively. To clarify the situation, and to make clear the required input to the recursion relations, we start by giving a recursive formula for the Fourier coefficients of normalized Hauptmodul $\phi$ of $\Gamma$. 
Applying Theorem 3.5 to the (normalized) derivative of the Hauptmodul $\phi$ gives the equation

$$
\begin{aligned}
-n c_{n}=\frac{1}{n+1} & \left\{S\left(n+1 ; 0,-c_{1},-2 c_{2}, \ldots,-(n-1) c_{n-1}\right)\right. \\
& -\frac{4 h_{0}}{\tau(N)} \sum_{\delta \mid N} \delta \sigma\left(\frac{n+1}{\delta h_{0}}\right)-\sum_{i=1}^{t}\left(1-\frac{h_{i} \sigma(N)}{6 \tau(N)}\right) F_{n+1}\left(\phi\left(s_{i}\right)\right) \\
& \left.-\sum_{\tau \in \Gamma \backslash \mathcal{H}}\left(1-e_{\tau}^{-1}\right) F_{n+1}(\phi(\tau))\right\} .
\end{aligned}
$$

As $c_{n}$ occurs linearly on each side of this equation, we can solve for $c_{n}$. The result, after simplification and applying Corollary 2.3, is as follows.

THEOREM 3.6. If $n=-1$, then $c_{-1}=1$.

If $n=0$, then

$$
\begin{aligned}
c_{0}= & \frac{24 h_{0}}{\sigma(N) h_{0}+6 \tau(N)} \delta_{1, h_{0}}+\frac{6 \tau(N)}{\sigma(N) h_{0}+6 \tau(N)} \\
& \times\left\{\sum_{i=1}^{t}\left(1-\frac{h_{i} \sigma(N)}{6 \tau(N)}\right) \phi\left(s_{i}\right)+\sum_{\tau \in \Gamma \backslash \mathcal{H}}\left(1-e_{\tau}^{-1}\right) \phi(\tau)\right\} .
\end{aligned}
$$

If $n>0$, then

$$
\begin{aligned}
& c_{n}=\frac{1}{(n+1)\left(1+\frac{h_{0} \sigma(N)}{6 \tau(N)}+n\right)} \\
& \times\left\{\frac{4 h_{0}}{\tau(N)} \sum_{\delta \mid N} \delta \sigma\left(\frac{n+1}{\delta h_{0}}\right)-S\left(n+1 ; 0,-c_{1}, \ldots,-(n-1) c_{n-1}\right)\right. \\
& +\sum_{i=1}^{t}\left(1-\frac{h_{i} \sigma(N)}{6 \tau(N)}\right) S\left(n+1 ; c_{0}-\phi\left(s_{i}\right), c_{1}, \ldots, c_{n-1}\right) \\
& \left.+\sum_{\tau \in \Gamma \backslash \mathcal{H}}\left(1-e_{\tau}^{-1}\right) S\left(n+1 ; c_{0}-\phi(\tau), c_{1}, \ldots, c_{n-1}\right)\right\} \text {. }
\end{aligned}
$$

Using Corollary 2.3, Theorem 3.5 can be rewritten to make the dependence on the coefficients of $\phi$ explicit.

THEOREM 3.7. Let the hypotheses be as in Theorem 3.5 and let $c_{i}, i=-1,0,1, \ldots$, be the coefficients of Hauptmodul $\phi$ of $\Gamma$, then 


$$
\begin{aligned}
a_{n}=\frac{1}{n} & \left\{S\left(n ; a_{1}, \ldots, a_{n-1}\right)-\frac{2 k h_{0}}{\tau(N)} \sum_{\delta \mid N} \delta \sigma\left(\frac{n}{\delta h_{0}}\right)\right. \\
& -\sum_{i=1}^{t}\left(\operatorname{ord}_{s_{i}} f-\frac{k h_{i} \sigma(N)}{12 \tau(N)}\right) S\left(n ; c_{0}-\phi\left(s_{i}\right), c_{1}, \ldots, c_{n-1}\right) \\
& \left.-\sum_{\tau \in \Gamma \backslash \mathcal{H}} \operatorname{ord}_{\tau} f S\left(n ; c_{0}-\phi(\tau), c_{1}, \ldots, c_{n-1}\right)\right\} .
\end{aligned}
$$

REMARK 3.8. By Theorems 3.6 and 3.7, the recurrence relations established require as input:

- $\quad$ a square-free integer $N$ such that $\Gamma$ is a subgroup of $\Gamma_{0}^{+}(N)$;

- the weight and divisor of $f$;

- the ramification locus and the ramification orders of the Hauptmodul $\phi_{\Gamma}$ and the values $\phi_{\Gamma}(\tau)$, where $\operatorname{ord}_{\tau} f \neq 0$.

Given this input, the relations have the same form for all $\Gamma$ and $f$ and are, in this sense, universal.

REMARK 3.9. More generally, suppose that $f(z)$ is a form of rational weight $k$ and multiplier $v$ on $\Gamma$, where $\Gamma$ is a subgroup of some $\Gamma_{0}^{+}(N)$ as above. If there is some integer $D$ such that $f^{D}(z)$ is a form of integral weight and trivial multiplier, then $f(z)$ has an expansion of the form (3.1) and we can apply the previous arguments to the quotient $f^{D \varepsilon} / h^{D k}$, where $h$ and $\varepsilon$ are as in Section 3.1. So in this case also the coefficients of $f(z)$ will satisfy the recurrences of Theorems 3.5 and 3.7.

\section{References}

[1] S. Ahlgren, 'The theta-operator and the divisors of modular forms on genus zero subgroups', Math. Res. Lett. 10(5-6) (2003), 787-798.

[2] D. Alexander, C. Cummins, J. McKay and C. Simons, 'Completely replicable functions', in: Groups, Combinatorics \& Geometry (Durham, 1990), London Mathematical Society Lecture Note Series, 165 (Cambridge University Press, Cambridge, 1992), pp. 87-98.

[3] J. R. Atkinson, 'Divisors of modular forms on $\Gamma_{0}(4)$ ', J. Number Theory 112(1) (2005), 189-204.

[4] R. E. Borcherds, 'Monstrous moonshine and monstrous Lie superalgebras', Invent. Math. 109(2) (1992), 405-444.

[5] A. Bouali, 'Faber polynomials, Cayley-Hamilton equation and Newton symmetric functions', Bull. Sci. Math. 130(1) (2006), 49-70.

[6] J. H. Bruinier, W. Kohnen and K. Ono, 'The arithmetic of the values of modular functions and the divisors of modular forms', Compositio Math. 140(3) (2004), 552-566.

[7] D. Choi, 'On values of a modular form on $\Gamma_{0}(N)$ ', Acta Arith. 121(4) (2006), 299-311.

[8] S. Y. Choi, 'The values of modular functions and modular forms', Canad. Math. Bull. 49(4) (2006), 526-535.

[9] J. H. Conway and S. P. Norton, 'Monstrous moonshine', Bull. Lond. Math. Soc. 11(3) (1979), 308-339.

[10] C. J. Cummins, 'Congruence subgroups of groups commensurable with PSL(2, $\mathbb{Z})$ of genus 0 and 1', Experiment. Math. 13(3) (2004), 361-382. 
[11] C. J. Cummins, 'On conjugacy classes of congruence subgroups of $\operatorname{PSL}(2, \mathbb{R})$ ', LMS J. Comput. Math. 12 (2009), 264-274.

[12] C. J. Cummins and T. Gannon, 'Modular equations and the genus zero property of moonshine functions', Invent. Math. 129(3) (1997), 413-443.

[13] D. Ford, J. McKay and S. P. Norton, 'More on replicable functions', Comm. Algebra 22(13) (1994), 5175-5193.

[14] H. Helling, 'Bestimmung der Kommensurabilitätsklasse der Hilbertschen Modulgruppe', Math. Z. 92 (1966), 269-280.

[15] H. Helling, 'On the commensurability class of the rational modular group', J. Lond. Math. Soc. (2) 2 (1970), 67-72.

[16] G. A. Jones, 'Congruence and noncongruence subgroups of the modular group: a survey', in: Proceedings of Groups-St. Andrews 1985, London Mathematical Society Lecture Note Series, 121 (Cambridge University Press, Cambridge, 1986), pp. 223-234.

[17] D. H. Lehmer, 'Properties of the coefficients of the modular invariant $J(\tau)$ ', Amer. J. Math. 64 (1942), 488-502.

[18] I. G. Macdonald, Symmetric Functions and Hall Polynomials, 2nd edn, Oxford Mathematical Monographs (Clarendon Press, Oxford, 1995); with contributions by A. Zelevinsky.

[19] K. Mahler, 'On a class of nonlinear functional equations connected with modular functions', J. Aust. Math. Soc. Ser. A 22(1) (1976), 65-118.

[20] M. Newman, 'Construction and application of a class of modular functions', Proc. Lond. Math. Soc. (3) 7 (1957), 334-350.

[21] M. Newman, 'Construction and application of a class of modular functions. II', Proc. Lond. Math. Soc. (3) 9 (1959), 373-387.

[22] S. P. Norton, 'More on moonshine', in: Computational Group Theory (Durham, 1982) (Academic Press, London, 1984), pp. 185-193.

[23] S. P. Norton, Private communication.

[24] G. Shimura, Introduction to the Arithmetic Theory of Automorphic Functions, Kanô Memorial Lectures, No. 1. Publications of the Mathematical Society of Japan, 11 (Iwanami Shoten, Tokyo and Princeton University Press, Princeton, NJ, 1971).

[25] P. K. Suetin, Series of Faber Polynomials, Analytical Methods and Special Functions, Vol. 1 (Gordon and Breach, Amsterdam, 1998); translated from the 1984 Russian original by E. V. Pankratiev.

\title{
C. J. CUMMINS, Department of Mathematics and Statistics, Concordia University, Montréal, Québec, Canada \\ e-mail: cummins@mathstat.concordia.ca
}

\author{
N. S. HAGHIGHI, Department of Mathematics and Statistics, Concordia University, \\ Montréal, Québec, Canada \\ e-mail: n_sabet@mathstat.concordia.ca
}

